\title{
LINKAGE IN HEXASOMIC INHERITANCE
}

\author{
J. H. BENNETT \\ Department of Genetics, Cambridge
}

Received 1.i.53

\section{INTRODUCTION}

IN studies on genetic linkage, the primary object is to form estimates of the frequencies of the modes of gamete formation from data on the gametic output of individuals of known genotype. With disomic inheritance one parameter, the recombination fraction, enters into the law of inheritance for pairs of linked loci and in the absence of differential viability, this can be estimated from the gametic output of either of the two types of double heterozygote. With polysomic inheritance, a multiplicity of segregating genotypes and of modes of gamete formation makes the analysis more complex.

\section{TETRASOMIC INHERITANCE AT TWO LINKED LOCI}

With two linked loci and tetrasomic inheritance, there are eleven modes of gamete formation. An example of this genetic situation has been discovered in Lythrum salicaria (Fisher, 1949). Fisher (1947) has considered this case in some detail. He has shown how, in general, the heterogenic genotypes can be classified into isomorphic sets such that all members of a set can be generated from any constituent member by systematic gene substitutions. It follows that genotypes which belong to the same set yield populations of gametes with equivalent frequencies. In accordance with a generalised form of Mendel's law, there is determined for any genotype, a gametic series for each mode of gamete formation. The gametic output is then expressible in the form of a matrix with the rows corresponding with the different modes of gamete formation and the columns with the different gametic genotypes. In most cases, the rank of the gametic matrix is less than the number of modes of gamete formation so that the gametic output from a particular isomorphic set of genotypes is deficient in some of the information sought. A detailed analysis is required in order to find out what information becomes available on considering genotypes from different isomorphic sets. The results of the analysis for two linked tetrasomic loci are summarised in fig. I, which is reproduced from a wall diagram in the Department of Genetics, Cambridge, by the kind permission of Sir Ronald Fisher.

With only two alleles at each of the two loci, the frequencies of all eleven modes of gamete formation cannot be estimated. However, 
six can be estimated as well as four independent linear combinations involving the other five.

Fisher's graphical analysis of the $\mathbf{2 4}$ gametic matrices in tetrasomic inheritance with loci

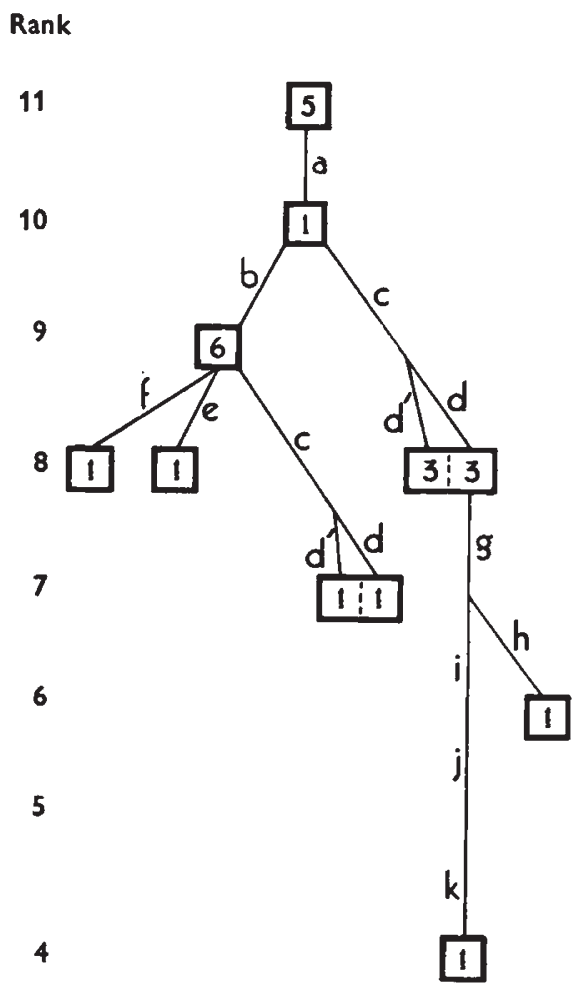

Deficiency components

(a) $1,-2 \cdot . \quad \cdot \quad \cdot \quad 2 \quad-1 \mid$

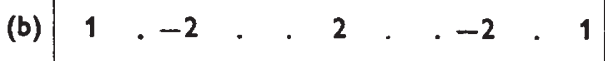

(c) $\begin{array}{lllllllllll} & 1 & -2 & -2 & -2 & 2 & 2 & -1 & 1\end{array}$

(d) $1 \quad \cdot \quad \cdot \begin{array}{lllllll}-2 & -2 & 2 & \cdot & \cdot & 1\end{array}$

(d') $1 \quad \cdot \quad \begin{array}{llllllll} & -2 & \cdot & -2 & \cdot & 2 & . & 1\end{array}$

(e)

e) $\cdot \quad \cdot \quad \cdot 1-1-1,1$

(f) $-1-1 \quad . \quad 2 \quad 2 \quad 1-1-1 \quad . \quad-1$

(g) $\cdot \quad \cdot 2-2 \cdot 2-2 \cdot . \cdot=1 d$

(h) $\cdot \quad \cdot \quad \cdot \quad \cdot 11-2-11$

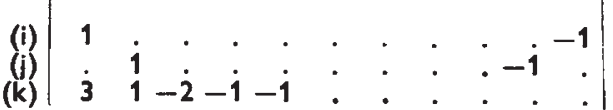

Fig. I.-Numbers of isomorphic sets having gametic matrices of different rank, with the particular components of deficiency in which they differ.

\section{HEXASOMIC INHERITANCE AT TWO LINKED LOCI}

It is of some interest to consider the problem presented by linkage between two loci showing hexasomic inheritance. Although hexasomic inheritance has not yet been demonstrated in any organism, it is not unexpected in autohexaploids.

It might be noted here that polyploids are conveniently classified both according to the origin of their germ plasm and according to their breeding behaviour. If polyploidy has originated from a cross involving only one species, the new individual is an auto-polyploid whereas if more than one species was involved in the cross, it is an allo-polyploid. Polyploids which on breeding always behave as diploids are properly called amphi-diploids. These are to be distinguished from polyploids which exhibit polysomic inheritance and in which, at meiosis, a gene has more than one possible partner. 
Genetical behaviour might not be of the same nature throughout the whole germ plasm and it is conceivable that inheritance might be polysomic in one set of chromosomes and disomic in another.

With two linked loci and hexasomic inheritance, there are forty modes of gamete formation. Using Fisher's enumeration (1947, p. 72) these are shown in table I where, for each mode of formation,

TABLE I

\begin{tabular}{|c|c|c|c|}
\hline $\begin{array}{l}\text { Mode of } \\
\text { formation }\end{array}$ & $\begin{array}{l}\text { Typical } \\
\text { gamete }\end{array}$ & $\begin{array}{l}\text { Mode of } \\
\text { formation }\end{array}$ & $\begin{array}{l}\text { Typical } \\
\text { gamete }\end{array}$ \\
\hline $\begin{array}{l}1 \\
2 \\
3 \\
4 \\
5 \\
6 \\
7 \\
8 \\
9 \\
10 \\
11 \\
12 \\
13 \\
14 \\
15 \\
16 \\
17 \\
18 \\
19 \\
20\end{array}$ & $\begin{array}{l}a_{1} b_{1} / a_{2} b_{2} / a_{3} b_{3} \\
a_{1} b_{1} / a_{1} b_{1} / a_{2} b_{2} \\
a_{1} b_{1} / a_{2} b_{2} / a_{3} b_{4} \\
a_{1} b_{1} / a_{2} b_{1} / a_{2} b_{2} \\
a_{1} b_{1} / a_{1} b_{1} / a_{2} b_{3} \\
a_{1} b_{1} / a_{2} b_{3} / a_{4} b_{3} \\
a_{1} b_{1} / a_{2} b_{3} / a_{2} b_{3} \\
a_{1} b_{1} / a_{2} b_{3} / a_{3} b_{2} \\
a_{1} b_{1} / a_{2} b_{3} / a_{3} b_{4} \\
a_{1} b_{2} / a_{2} b_{2} / a_{2} b_{3} \\
a_{1} b_{1} / a_{1} b_{2} / a_{2} b_{1} \\
a_{1} b_{2} / a_{3} b_{4} / a_{3} b_{3} \\
a_{1} b_{2} / a_{1} b_{2} / a_{3} b_{4} \\
a_{1} b_{2} / a_{2} b_{1} / a_{3} b_{4} \\
a_{1} b_{2} / a_{3} b_{2} / a_{3} b_{4} \\
a_{1} b_{2} / a_{3} b_{1} / a_{3} b_{2} \\
a_{1} b_{2} / a_{2} b_{1} / a_{2} b_{1} \\
a_{1} b_{2} / a_{2} b_{3} / a_{4} b_{3} a_{1} b_{2} / a_{2} b_{3} / a_{3} b_{4} a_{1} b_{2} / a_{2} b_{3} / a_{3} b_{1}\end{array}$ & $\begin{array}{l}21 \\
22 \\
23 \\
24 \\
25 \\
26 \\
27 \\
28 \\
29 \\
30 \\
31 \\
32 \\
33 \\
34 \\
35 \\
36 \\
37 \\
38 \\
39 \\
40\end{array}$ & $\begin{array}{l}a_{1} b_{1} / a_{2} b_{2} / a_{2} b_{3} \\
a_{1} b_{1} / a_{2} b_{2} / a_{3} b_{2} \\
a_{1} b_{1} / a_{2} b_{3} / a_{2} b_{4} \\
a_{1} b_{1} / a_{3} b_{2} / a_{4} b_{2} \\
a_{1} b_{1} / a_{1} b_{2} / a_{3} b_{4} \\
a_{1} b_{1} / a_{2} b_{1} / a_{4} b_{3} \\
a_{1} b_{1} / a_{1} b_{2} / a_{3} b_{2} \\
a_{1} b_{1} / a_{2} b_{1} / a_{2} b_{3} \\
a_{1} b_{1} / a_{1} b_{2} / a_{2} b_{3} \\
a_{1} b_{1} / a_{2} b_{1} / a_{3} b_{2} \\
a_{1} b_{2} / a_{1} b_{3} / a_{4} b_{6} \\
a_{2} b_{1} / a_{3} b_{1} / a_{3} b_{4} \\
a_{1} b_{2} / a_{1} b_{3} / a_{3} b_{4} \\
a_{2} b_{1} / a_{3} b_{1} / a_{4} b_{3} \\
a_{1} b_{2} / a_{1} b_{3} / a_{4} b_{1} \\
a_{2} b_{1} / a_{3} b_{1} / a_{1} b_{4} \\
a_{1} b_{2} / a_{1} b_{3} / a_{2} b_{1} \\
a_{2} b_{1} / a_{3} b_{1} / a_{1} b_{2} \\
a_{1} b_{2} / a_{1} b_{2} / a_{2} b_{3} \\
a_{2} b_{1} / a_{2} b_{1} / a_{3} b_{2}\end{array}$ \\
\hline
\end{tabular}

there is given the genotype of a typical gamete that would arise from the parental genotype $a_{1} b_{1} / a_{2} b_{2} / a_{3} b_{3} / a_{4} b_{4} / a_{5} b_{5} / a_{6} b_{6}$.

There are sixty hexasomic genotypes with two alleles at each of

TABLE 2

Numbers of isomorphic sets of digenic hexasomic genotypes classified according to the partitions at two loci

\begin{tabular}{|c|c|c|c|}
\hline Partitions & $(5 \mathrm{I})$ & $\left(4^{2}\right)$ & $\left(3^{2}\right)$ \\
\hline$\left(5^{1}\right)$ & 2 & 2 & 1 \\
$\left(4^{2}\right)$ & 2 & 3 & 2 \\
$\left(3^{2}\right)$ & 1 & 2 & 2 \\
\hline
\end{tabular}

two linked loci and these fall into seventeen isomorphic sets of which seven are self-conjugate, the remainder comprising five conjugate pairs. These sets can be classified as shown in table 2 according to the partitions at the two loci.

The number of gametic genotypes to which any parental genotype 
can give rise is determined by the partitions at both loci. These numbers are shown in table 3 .

TABLE 3

Numbers of gametic genotypes to which any parental genotype can lead

\begin{tabular}{|c|c|c|c|}
\hline Partitions & $(51)$ & $(42)$ & $\left(3^{2}\right)$ \\
\cline { 1 - 3 }$(51)$ & 13 & 16 & 16 \\
$(42)$ & 16 & 20 & 20 \\
$\left(3^{2}\right)$ & 16 & 20 & 20 \\
\hline
\end{tabular}

\section{THE GAMETIC MATRICES}

Since parental genotypes belonging to the same isomorphic set have equivalent gametic matrices and as each of the gametic matrices for two conjugate isomorphic sets can readily be developed from the other, it is sufficient if we construct gametic matrices for one parental genotype from each of the seven self-conjugate sets and from one of each pair of conjugate sets (see tables 5-16).

As the rank of any one of these gametic matrices cannot exceed two less than the number of gametic genotypes which enter into it, no parental genotype used alone can lead to estimates of more than eighteen independent linear combinations of the forty frequencies. The actual values of the ranks of the twelve gametic matrices are shown, with typical parental genotypes, in table 4 .

TABLE 4

Ranks of gametic matrices

\begin{tabular}{|c|c|c|c|c|c|}
\hline & $(5 x)$ & & $(42)$ & & $\left(3^{2}\right)$ \\
\hline$(51)$ & $\begin{array}{l}A B / a b|a b| a b|a b| a b \\
A b|a B| a b|a b| a b \mid a b\end{array}$ & $\begin{array}{l}\text { II } \\
\text { II }\end{array}$ & $\begin{array}{l}A b / A b / a B / a b / a b / a b: \\
A B / A b / a b / a b / a b / a b:\end{array}$ & $\begin{array}{l}13 \\
13\end{array}$ & $A B / A b / A b / a b / a b / a b:$ II \\
\hline$(42)$ & $\ldots$ & & $\begin{array}{l}A B|A B| a b|a b| a b \mid a b: \\
A B|A b| a B|a b| a b \mid a b: \\
A b|A b| a B|a B| a b \mid a b:\end{array}$ & $\begin{array}{l}16 \\
16 \\
16\end{array}$ & $\begin{array}{l}A B / A B / A b / a b / a b / a b: \\
A B / A b / A b / a B / a b / a b:\end{array}$ \\
\hline$\left(3^{2}\right)$ & $\ldots$ & & $\cdots$ & & $\begin{array}{ll}A B / A B / A B / a b / a b / a b: & 10 \\
A B / A B \mid A b / a B / a b / a b: & 10\end{array}$ \\
\hline
\end{tabular}

Beneath each gametic matrix there is indicated a series of gametes whose classification would lead to the maximum number of independent linear combinations available for estimation from any parental genotype which belongs to the given isomorphic set. The number of gametes in any such series is, of course, equal to the rank of the gametic matrix. To take an example, the self-conjugate set of four genotypes which includes bisimplex coupling $(A B)(a b)_{5}$, can yield estimates of eleven independent linear combinations and at the bottom of table 5 there is indicated a series of eleven columns. In all, 147 columns are marked out in this way in the tables 5-16. 
TABLE 5

Parental genotype $\mathrm{AB} / \mathrm{ab} / \mathrm{ab} / \mathrm{ab} / \mathrm{ab} / \mathrm{ab}$, rcpresenting a set of 4 . Row divisor : 6

Composition of gamete

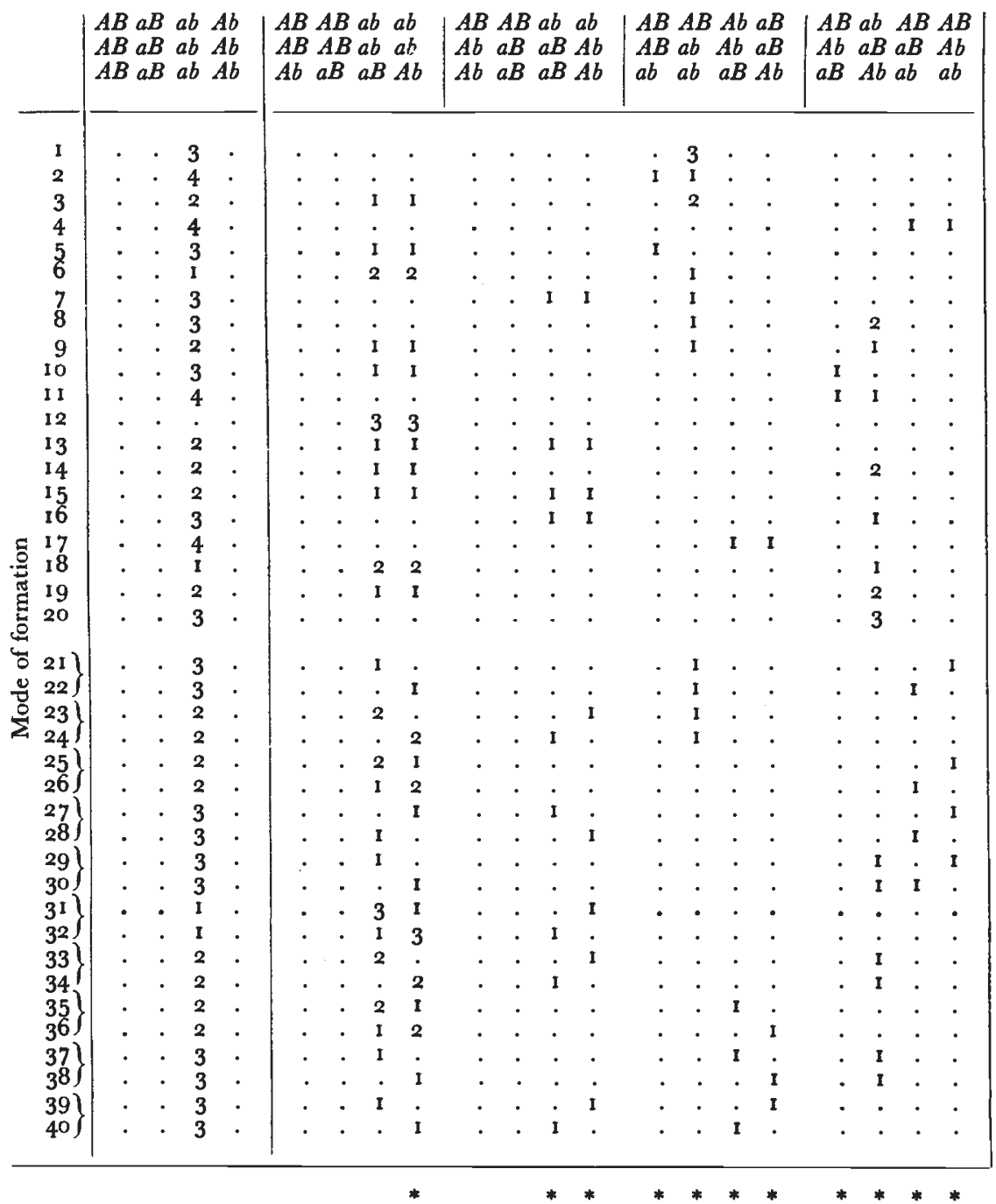


TABLE 6

Parental genotype $\mathrm{Ab} / \mathrm{aB} / \mathrm{ab} / \mathrm{ab} / \mathrm{ab} / \mathrm{ab}$, representing a set of 4 . Row divisor 30

Composition of gamete

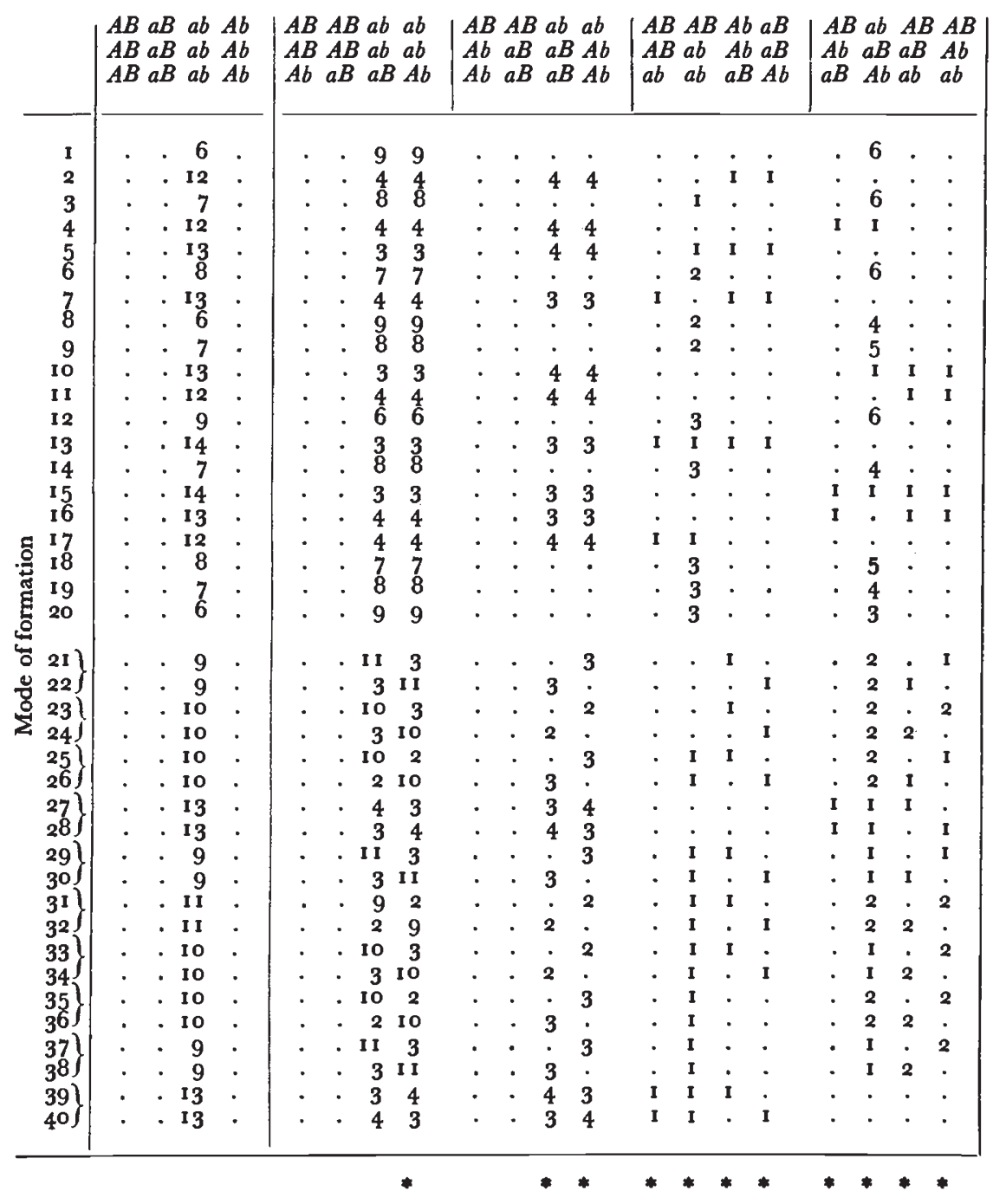




\section{TABLE 7}

Parental genotype $\mathrm{AB} / \mathrm{AB} / \mathrm{ab} / \mathrm{ab} / \mathrm{ab} / \mathrm{ab}$, representing a set of 4 . Row divisor : 15

Composition of gamete

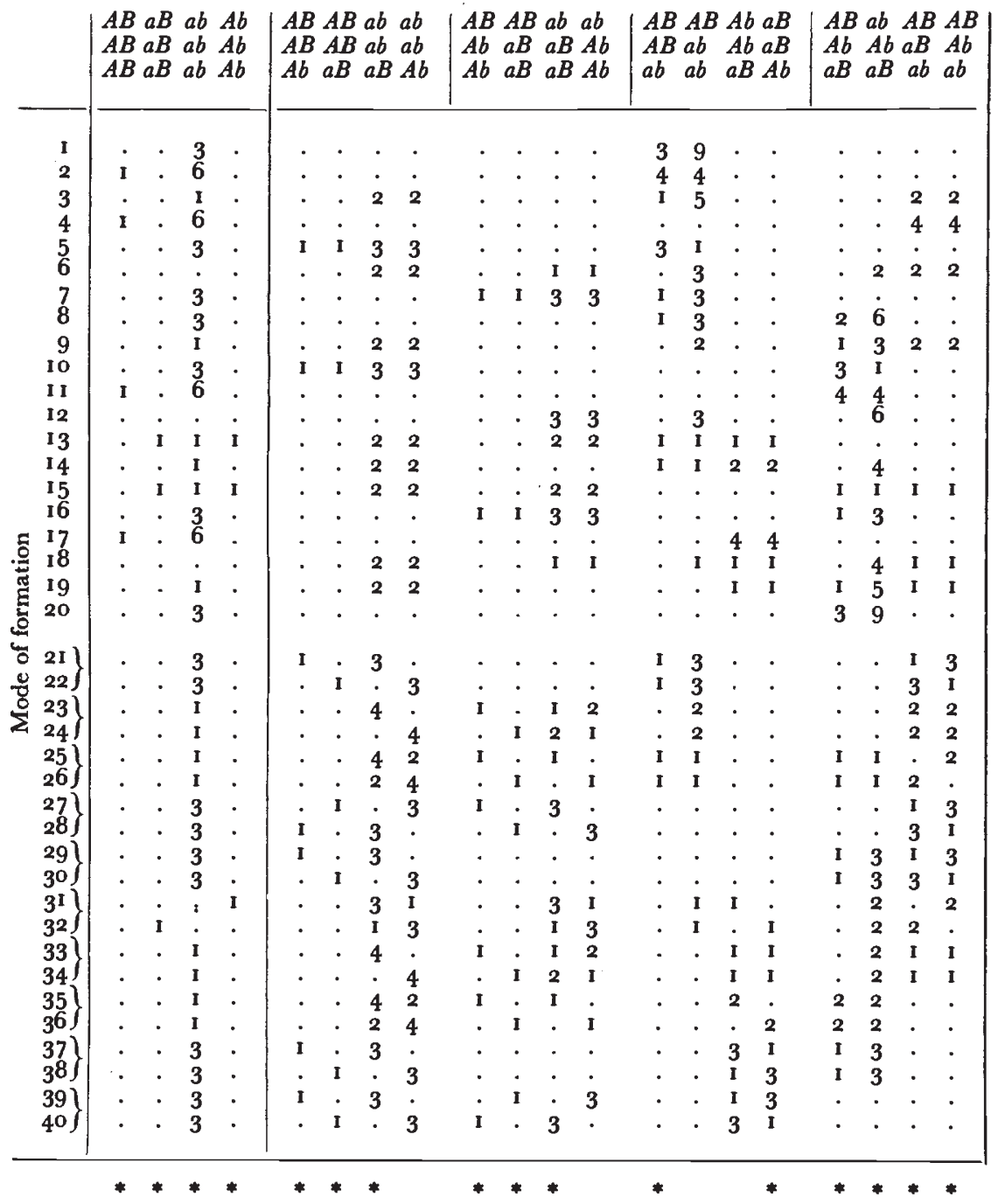




\section{TABLE 8}

Parental genotype $\mathrm{Ab} / \mathrm{Ab} / \mathrm{aB} / \mathrm{aB} / \mathrm{ab} / \mathrm{ab}$, representing a set of 4 .

Row divisor : 90

\section{Composition of gamete}

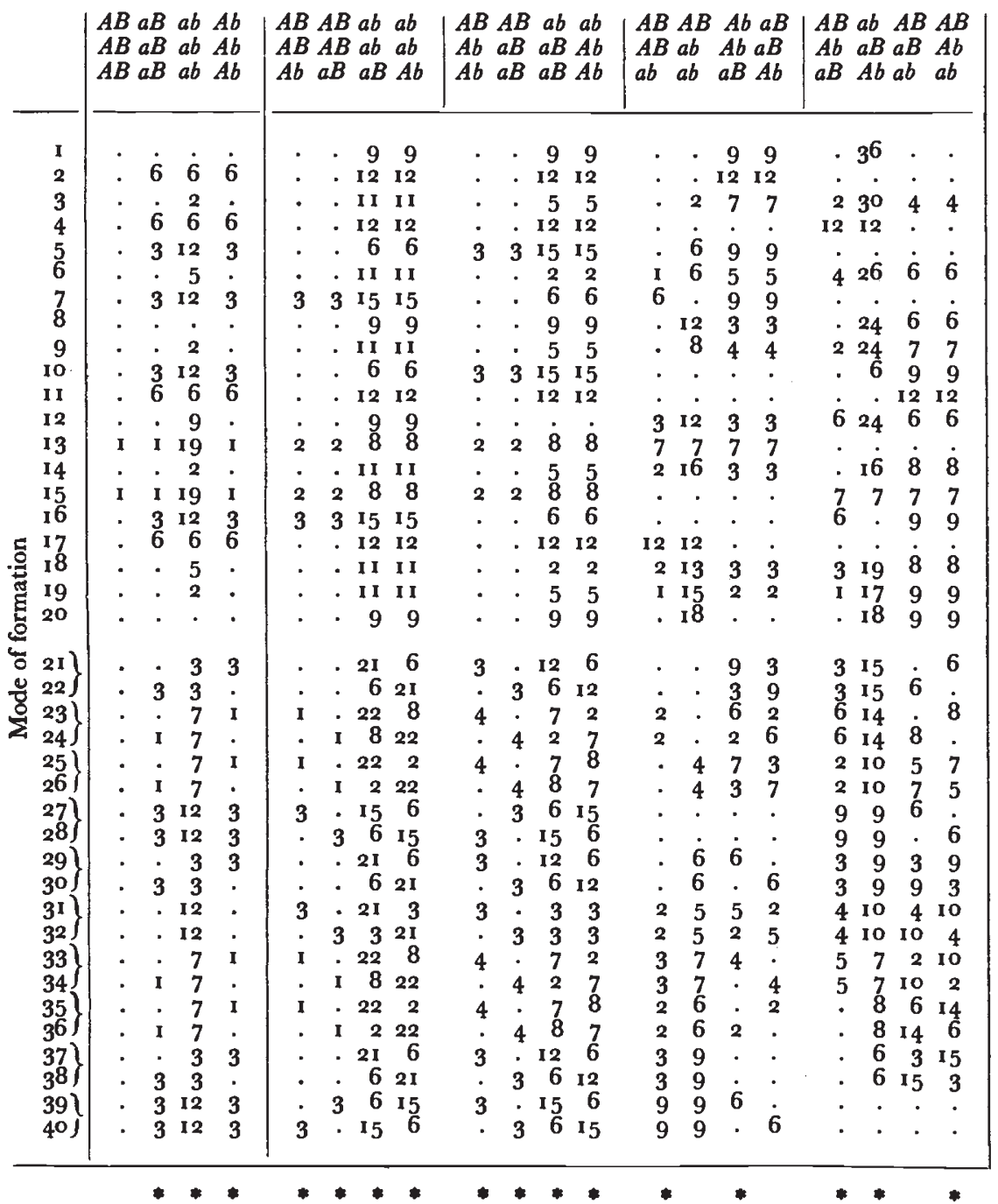




\section{TABLE 9}

Parental genotype $\mathrm{AB} / \mathrm{Ab} / \mathrm{aB} / \mathrm{ab} / \mathrm{ab} / \mathrm{ab}$, representing a set of 4 . Row divisor : 120

\section{Composition of gamete}

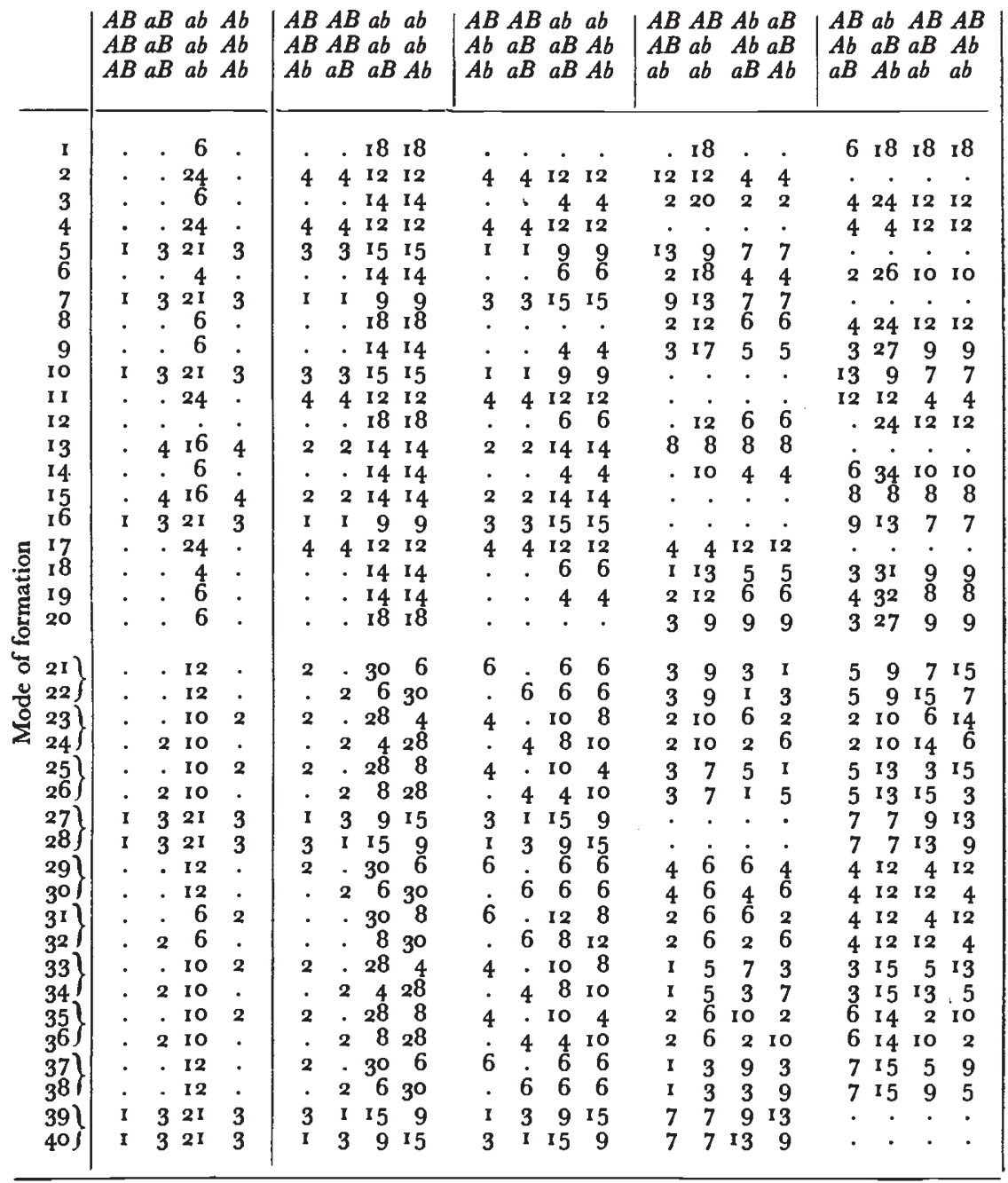

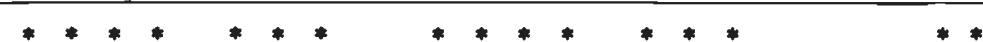




\section{TABLE 10}

Parental genotype $\mathrm{AB} / \mathrm{AB} / \mathrm{AB} / \mathrm{ab} / \mathrm{ab} / \mathrm{ab}$, representing a set of 2.

Row divisor : 20

Composition of gamete

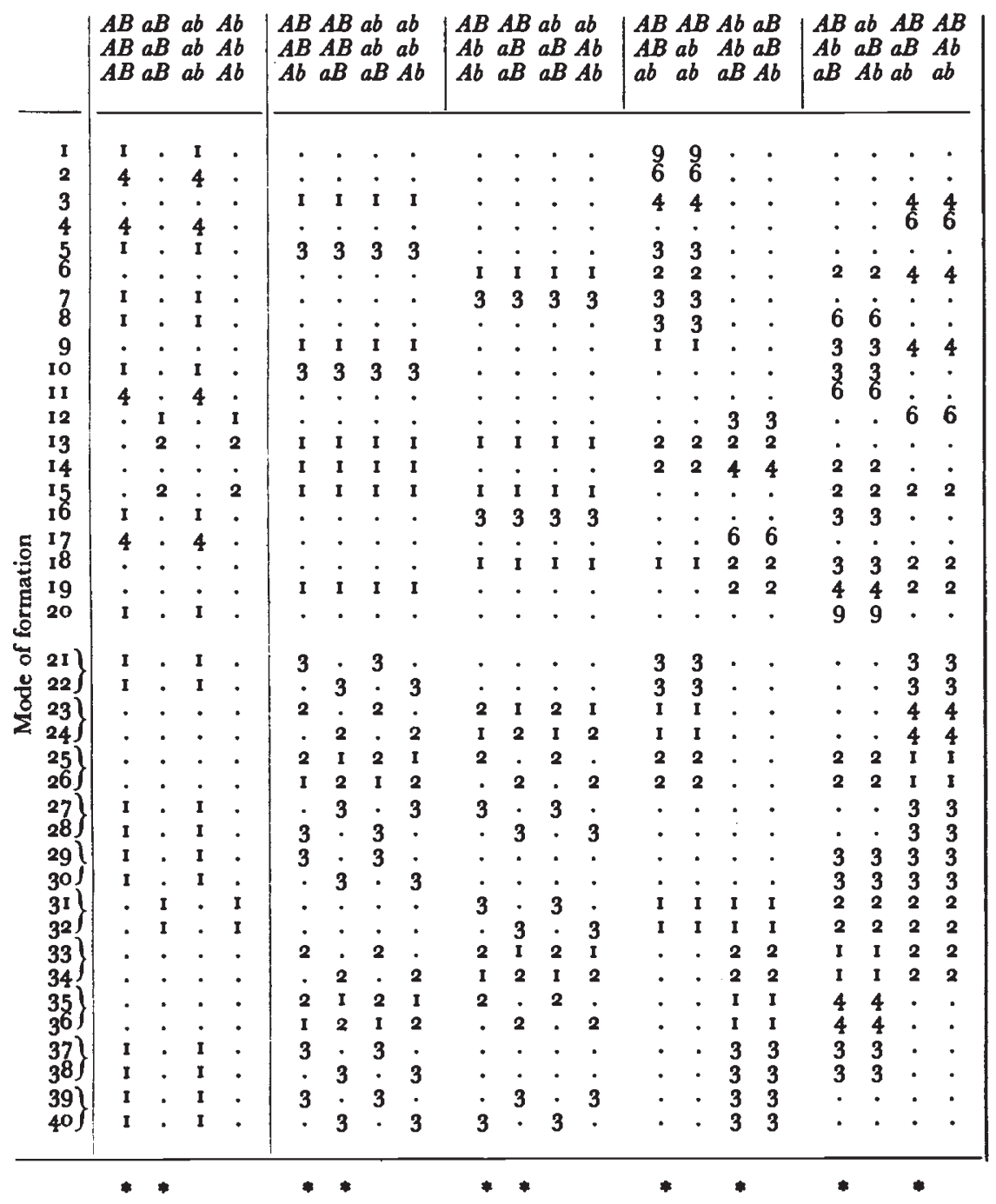


TABLE II

Parental genotype $\mathrm{AB} / \mathrm{AB} / \mathrm{Ab} / \mathrm{aB} / \mathrm{ab} / \mathrm{ab}$, representing a set of 2.

Row divisor : 180

Composition of gamete

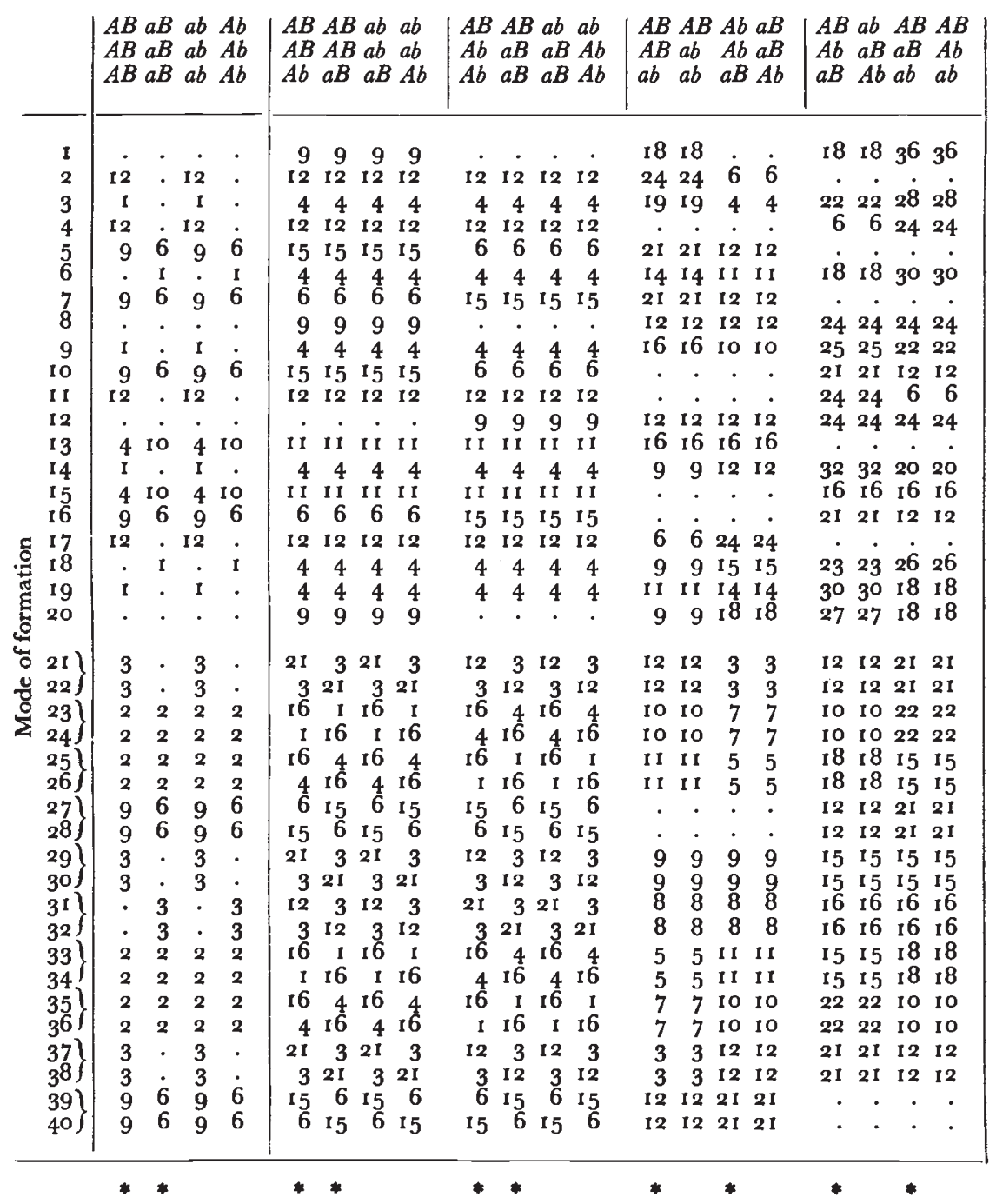




\section{TABLE 12}

Parental genotype $\mathrm{Ab} / \mathrm{Ab} / \mathrm{aB} / \mathrm{ab} / \mathrm{ab} / \mathrm{ab}$, representing a set of $4 . \quad$ An interchange of loci accompanied by an interchange of paired conjugate modes generates a conjugate set of 4 . Row divisor : 60

\section{Composition of gamete}

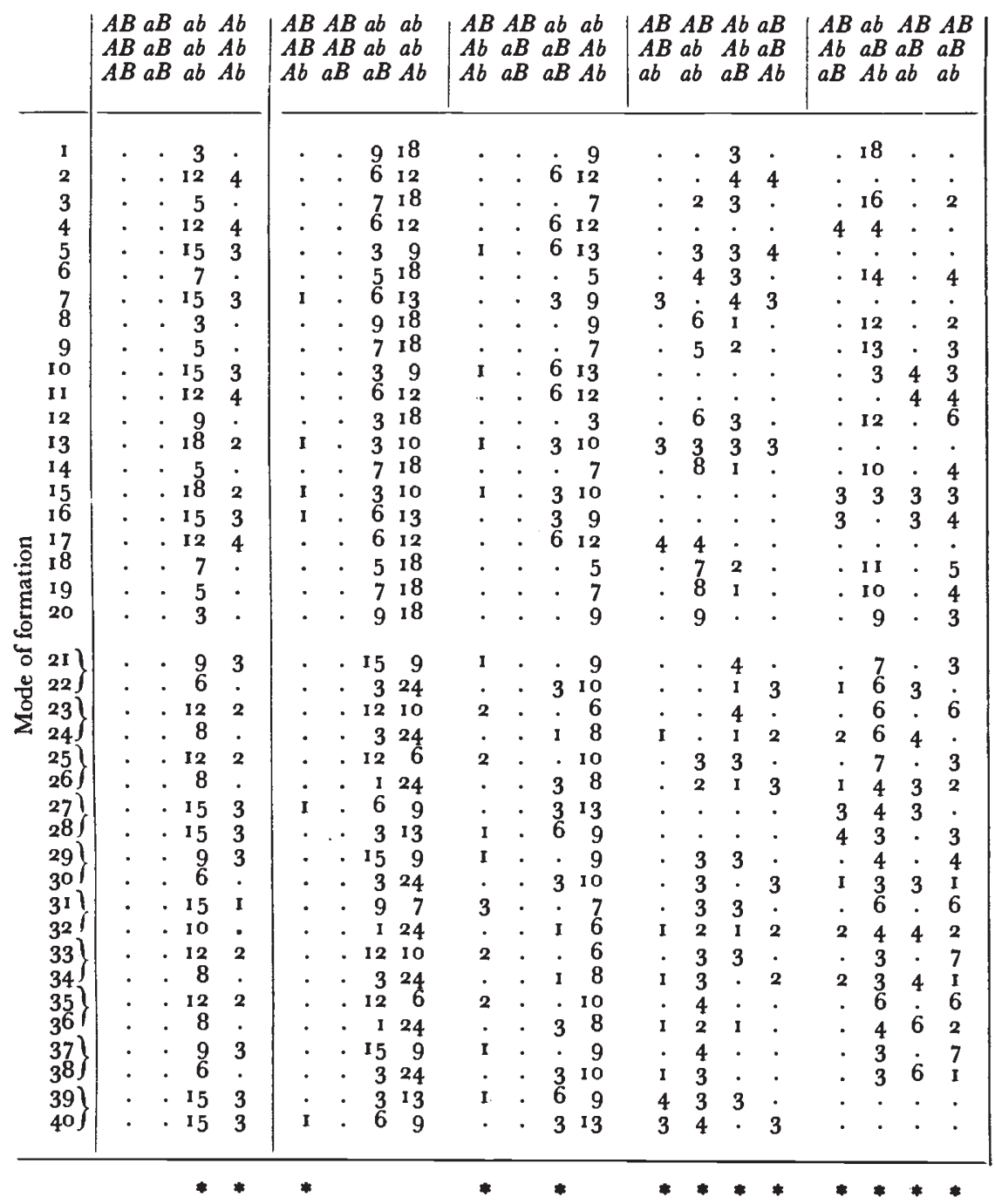




\section{TABLE 13}

Parental genotype $\mathrm{AB} / \mathrm{Ab} / \mathrm{ab} / \mathrm{ab} / \mathrm{ab} / \mathrm{ab}$, representing a set of $4 . \quad$ An interchange of loci accompanicd by an interchange of paired conjugate modes generates a conjugate set of 4 . Row divisor : 30

Composition of gamete

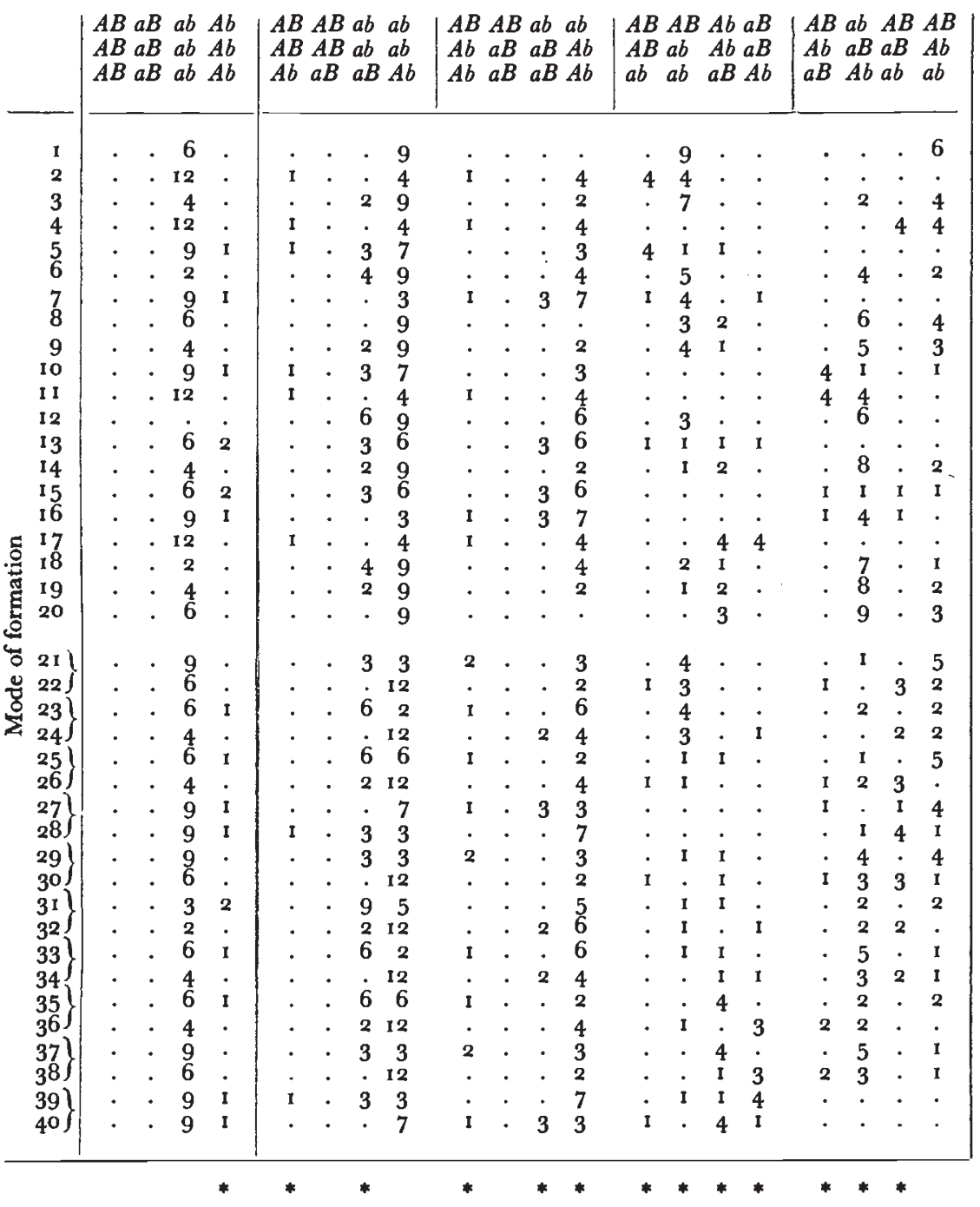




\section{TABLE 14}

Parental genotype $\mathrm{AB} / \mathrm{Ab} / \mathrm{Ab} / \mathrm{ab} / \mathrm{ab} / \mathrm{ab}$, representing a set of $4 . \quad$ An interchange of loci accompanied by an interchange of paired conjugate modes generates a conjugate set of 4 . Row divisor : 60

\section{Composition of gamete}

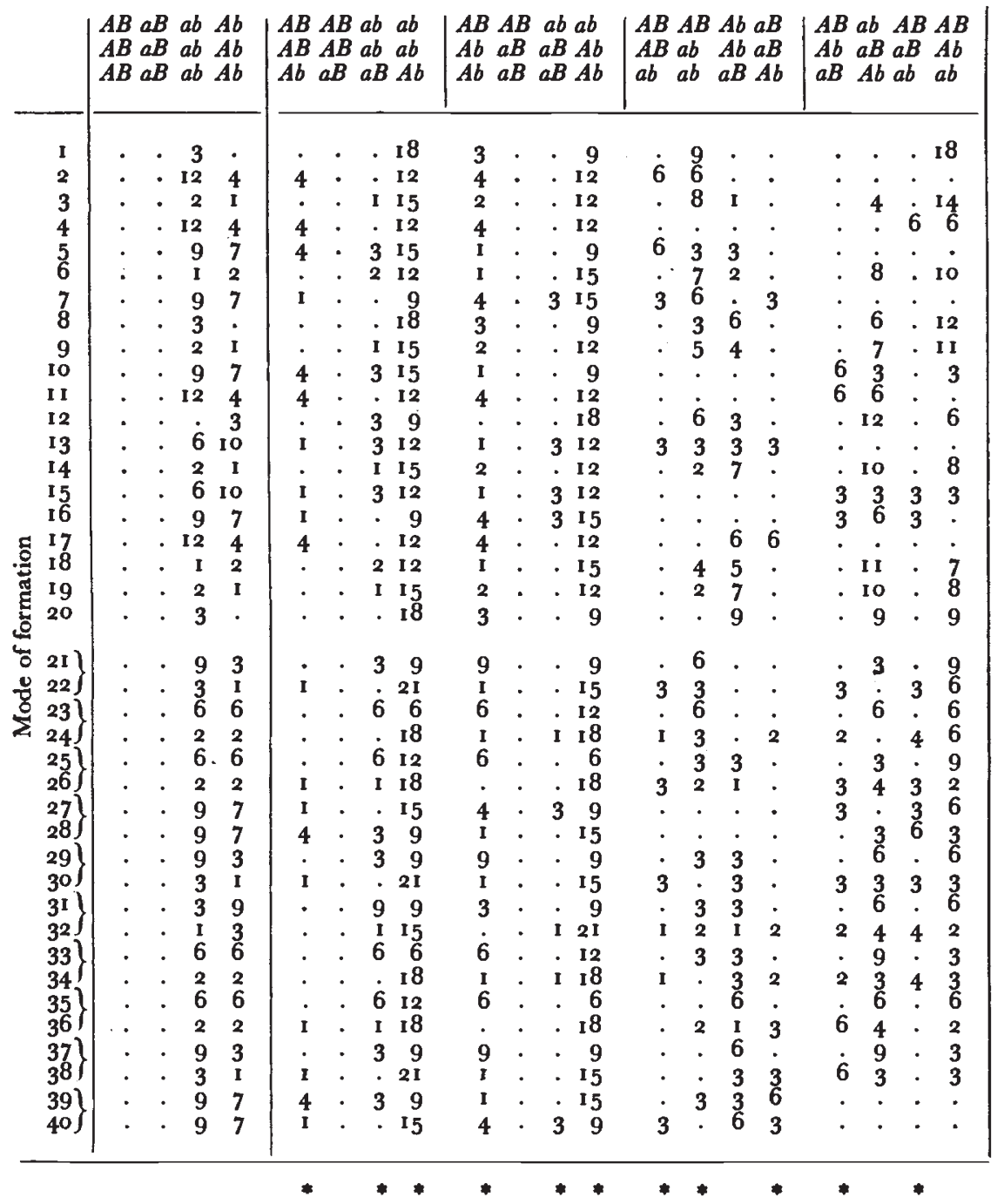




\section{TABLE 15}

Parental genotype $\mathrm{AB} / \mathrm{AB} / \mathrm{Ab} / \mathrm{ab} / \mathrm{ab} / \mathrm{ab}$, representing a set of $4 . \quad$ An interchange of loci accompanied by an interchange of paired conjugate modes generates a conjugate set of 4 . Row divisor : 60

\section{Composition of gamete}

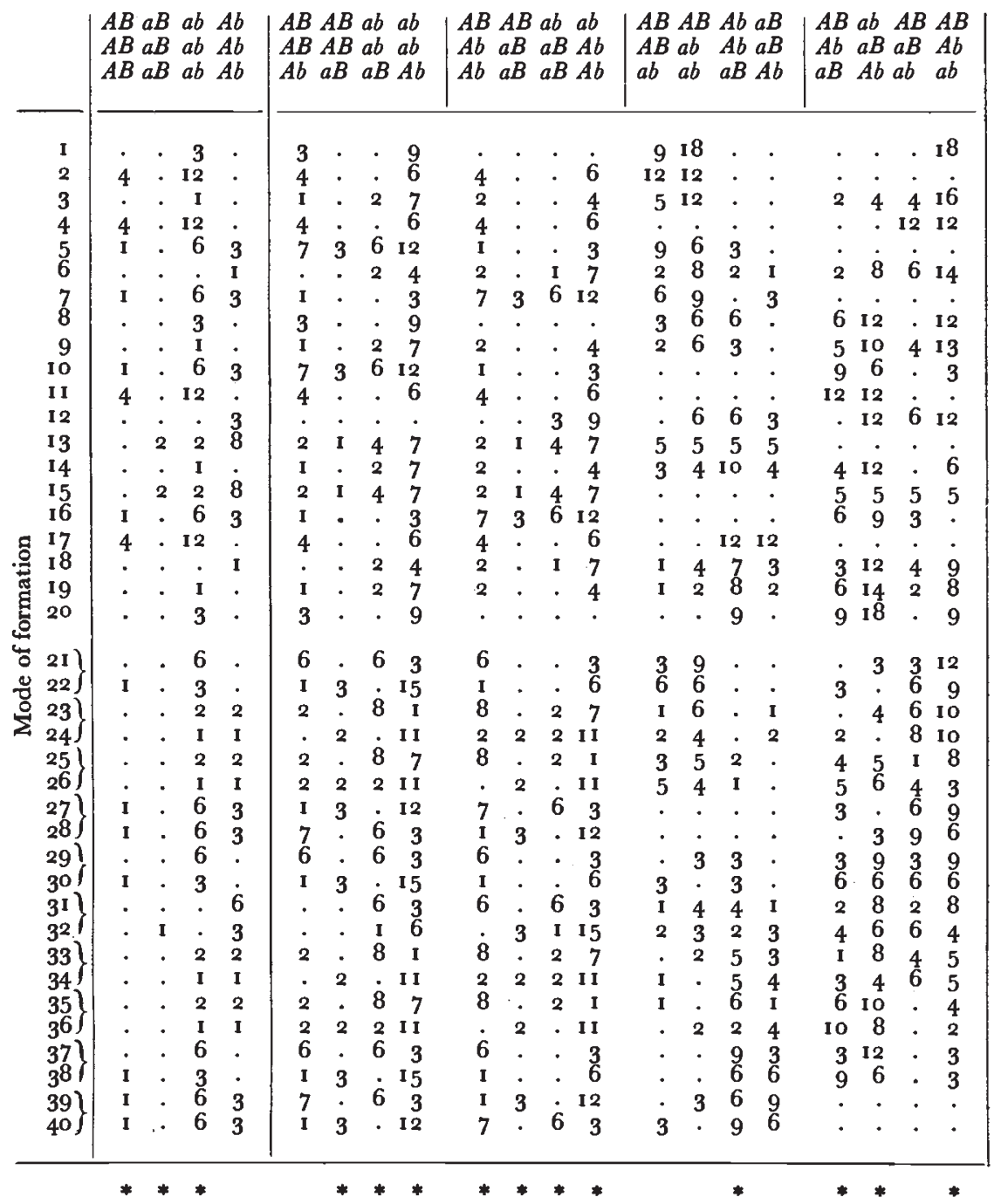


TABLE 16

Parental genotype $\mathrm{AB} / \mathrm{Ab} / \mathrm{Ab} / \mathrm{aB} / \mathrm{ab} / \mathrm{ab}$, representing a set of 2. An interchange of loci accompanied by an interchange of paired conjugate modes generates a conjugate set of 2. Row divisor : 180

\section{Composition of gamete}

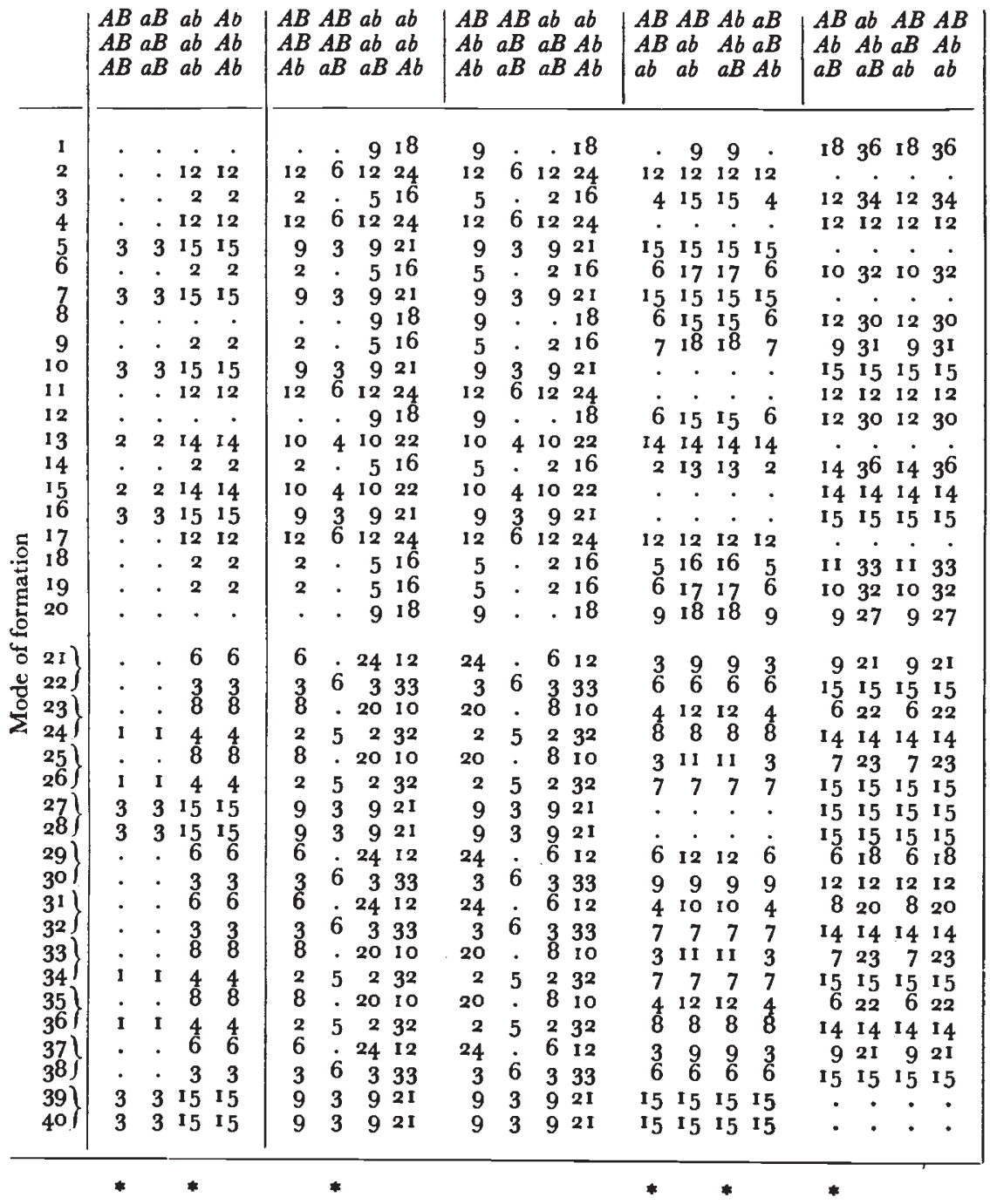


When we take account of the gametic matrices for the five conjugate sets of genotypes (which can be developed from the matrices of tables I 2-16 by an interchange of loci and of pairs of conjugate modes) there are 204 such columns. The rank of this $40 \times 204$ matrix is equal to the number of independent linear combinations of the forty frequencies which become available for estimation on classifying the gametic output of hexasomic organisms with two alleles at each of two linked loci.

Among the 147 columns marked out in the twelve gametic matrices, there are only twenty-four that are independent. Moreover, the gametic matrices for the five conjugate sets of genotypes contribute

\section{TABLE 17}

Linear combinations capable of estimation

Column reference numbers

\begin{tabular}{|c|c|c|c|c|c|c|c|c|c|c|c|c|c|c|c|c|c|c|c|c|c|c|c|c|}
\hline & I & 2 & 3 & 4 & 5 & 6 & 7 & & 9 & & & & 13 & & & & & & & & & 2 & & 24 \\
\hline & . & . & . & . & 3 & & . & & . & & & . & . & & . & & $\cdot$ & & & & . & . & & \\
\hline 2 & . & . & . & I & I & & . & • & & & . & $0^{\circ}$ & . & I & . & & 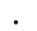 & . & . &. & • & . & & \\
\hline 3 & I & . & . & . & 2 & & ${ }^{\circ}$ & $\cdot$ & . & 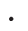 & . & . & . & . & . & $\cdot$ & . & 2 & . & . & . & . & & \\
\hline 4 & & . & . & . & . & . & 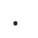 & . & . & I & I & . & I & I & . & . & . & . & . & . & . & . & . & \\
\hline 5 & I & . & . & I & . & . & . & . & . & - & . & . & . & . & . & . & I & 3 & . & . & . & . & & . \\
\hline & 2 & . & . & . & I & & . & · & . & . & . & . & . & . & . & . & & 2 & . & . & . & . & & \\
\hline 7 & . & I & I & . & I & . & . & . & . & . & . & I & . & 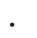 & . & . & . & . & . & . & & 3 & 3 & . \\
\hline 8 & & . & . & . & I & . & . & . & 2 & & . & 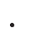 & . & 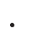 & . & . & . & . & & 2 & . & & & \\
\hline 9 & I & . & . & . & I & . & . & . & 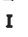 & 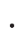 & . & . & . & . & . & . & . & 2 & & I & . & . & . & . \\
\hline 10 & I & . & . & . & . & . & . & I & $\cdot$ & - & . & . & . & . & . & . & I & 3 & . & 3 & . & . & . & \\
\hline I I & & . & . & . & . & . & . & I & I & . & . & . & . & I & . & . & . & 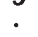 & . & 4 & . & . & & . \\
\hline 12 & 3 & . & . & . & . & . & . & . & . & . & . & . & . & . & . & . & . & . & . & . & . & . & & I \\
\hline I3 & I & I & I & . & . & . & . & . & . & . & . & I & . & . & I & I & . & 2 & I & . & I & 2 & 2 & \\
\hline I 4 & I & & . & . & . & . & . & . & 2 & . &. & . & & 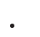 & . & & . & 2 & 2 & . & & . & & \\
\hline I 5 & I & I & I & - & . & . & . & 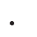 & 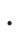 & 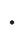 & 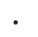 & . & I & . & I & I & . & 2 & & I & I & 2 & 2 & \\
\hline \& 16 & . & I & I & . & . & . & . & . & I & & . & . & I & . & . & . & . & . & . & I & . & 3 & 3 & \\
\hline I7 7 & . & . & . & . & . & I & I & . & . & . & . & I & . & I & . & . & . & . & 4 & . & . & & & \\
\hline 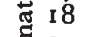 & 2 & . & . & . & . & . & . & . & I & 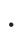 & . & . & . & 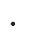 & . & . & & 2 & $\begin{array}{l}4 \\
\text { I }\end{array}$ & & . & : & : & \\
\hline ह & I & . & . & . & . & . & . & . & 2 & . & . & . & . & . & . & . & . & 2 & I & I & . & . & . & \\
\hline 20 & 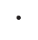 & . & . & $\cdot$ & . & $\cdot$ & $\cdot$ & $\cdot$ & 3 & - & $\cdot$ & $\cdot$ & . & . & . & . & . & . & . & 3 & . & & . & \\
\hline $2 \mathrm{I}$ & & . & . & . & I & . & . & . & . & 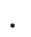 & t & . &. & . & . & & I & 3 & . & . & . & . & & \\
\hline 22 & I & . & . & . & I & . & . & . &. & I & 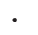 & . & . & . & . & . & . & & . & . & . & . & & \\
\hline 23 & & : & I & $\cdot$ & I & . & - & • & . & & . & 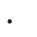 & . & . & . & . & & 4 & . & . & . & I & & \\
\hline 24 & 2 & I & . & . & I & . & . & . & . & • & • & . & . & . & . & . & . & & . & . & . & . & I & \\
\hline 25 & I & . & . & . & $\cdot$ & . & $\cdot$ & • & $\cdot$ & . & I & $\cdot$ & $\cdot$ & . & . & . & & 4 & & I & & I & & . \\
\hline 26 & 2 & 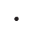 & . & . & . & . & . & . & $\cdot$ & I & 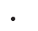 & . & . & & . & & & 2 & & I & . & & & \\
\hline & I & I & 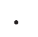 & . & . & . & 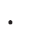 & . & . & & I & 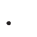 & I & & . & . &. & & $\therefore$ & & & 3 & & \\
\hline 28 & 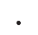 & . & I & . & . & . & . & . & . & I &. & . & I & . & . & & I & 3 & . & . & $\cdot$ & & 3 & \\
\hline 29 & & . & . & . & . & . & . & . & I & & 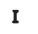 & & . & & . & & I & 3 & & I & & . & & \\
\hline & I & . & & . & . & . & 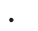 & . & I & I & & . & . & & 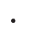 & 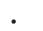 & 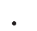 & & & & & & & \\
\hline 3 & I & . & I & 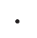 & . & . & . & 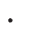 & . & & . & 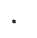 & . & . & . & I & . & 3 & & & . & 3 & & \\
\hline $3^{2}$ & 3 & I & $\cdot$ & . & $\cdot$ & . & $\cdot$ & . & . & & . & $\cdot$ & $\cdot$ & • & I & & . & I & I & $\cdot$ & . & & & \\
\hline & & & I & & & & & & I & & & 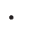 & & & & & & 4 & & & & I & & \\
\hline 34 & 2 & I & . & $\theta^{\prime}$ & . & 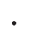 & . & . & I & & . & . & . & . & . & $\theta^{\circ}$ & . & $\boldsymbol{I}$ & I & 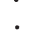 & . & & & \\
\hline 35 & I & . & . & . & . & I & & . & . & . & . & . & . & . & . & . & . & & . & 2 & . & I & & \\
\hline $3^{6}$ & 2 & . & . & . & . & • & I & . & 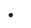 & & . & . & . & . & . & . & . & 2 & 2 & 2 & . & & & \\
\hline & & 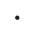 & . & . & . & I & . & & I & & & . & & & & & & 3 & & & & & & \\
\hline 3 & I & & & . & 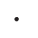 & 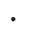 & I & . & I & 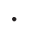 & . & . & 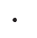 & & . & . & . & & 3 & I & . & . & & \\
\hline 39 & . & 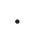 & $\dot{\mathbf{I}}$ & . & . & . & I & . & . & - & . & I & . & . & . & & I & 3 & 3 & . & . & & 3 & \\
\hline 40 & I & I & & . & & I & . & & & & . & I & . & . & . & . & . & & I & . & . & 3 & & . \\
\hline
\end{tabular}


no further independent combination. It happens that estimates of these twenty-four linear combinations can be formed from the gametic output of genotypes belonging only to self-conjugate isomorphic sets. The following example illustrates how this can be done. The selfconjugate set of genotypes which includes bisimplex coupling $(A B)(a b)_{5}$, is competent to estimate the eleven linear combinations shown in the first eleven columns of table i 7 . The self-conjugate set of four genotypes which includes bisimplex repulsion $(A b)(a B)(a b)_{4}$, also leads to estimates of eleven independent linear combinations, but of these, nine are held in common with those of the former set. The two independent combinations are shown in columns $\mathrm{I} 2$ and $\mathrm{I} 3$ of table $\mathrm{I} 7$. The self-conjugate set of four genotypes which includes biduplex coupling $(A B)_{2}(a b)_{4}$, contributes the seven independent combinations shown in columns I4-20, whilst the self-conjugate set of four genotypes which includes biduplex repulsion $(A b)_{2}(a B)_{2}(a b)_{2}$, adds three further columns (2I-23). The self-conjugate set of four genotypes which includes biduplex sub-coupling $(A B)(A b)(a B)(a b)_{3}$, * yields no linear combination that is independent of the first twenty-three columns of table 17. The remaining linear combination, shown in column 24, is found in the gametic matrix for the self-conjugate pair of genotypes one of which is bitriplex coupling $(A B)_{3}(a b)_{3}$. The progression in rank of the combined gametic matrices is shown in table 18 .

TABLE 18

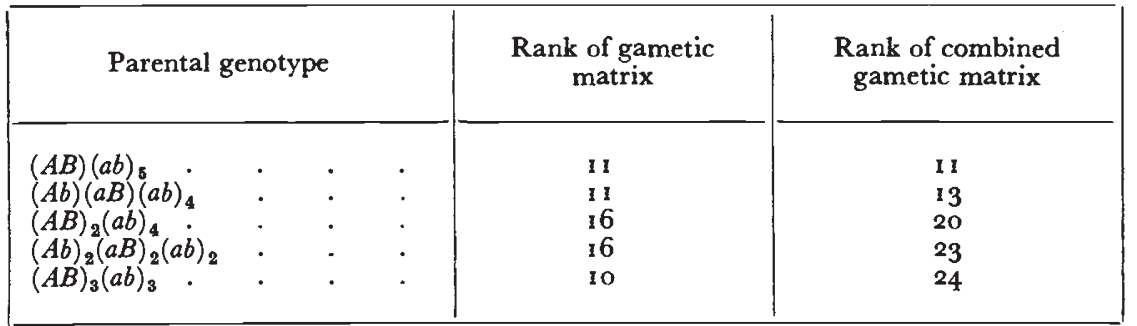

The $40 \times 16$ deficiency matrix is shown in table 19 . It is seen that the frequencies of only three modes of gamete formation, namely I 2, 31 and 32 can be estimated singly. This deficiency matrix merits further consideration as it must contain the essentials of a system of classification of the modes of gamete formation.

\section{SUMMARY}

Gametic matrices are given for twelve isomorphic sets of hexasomic genotypes with two alleles at each of two linked loci. It is found that from a classification at two linked loci of the gametic output from digenic hexasomic organisms, we can estimate only twenty-four

* There are four neutral genotypes. These are duplo-triplex and comprise a conjugate

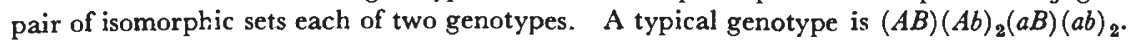


independent linear combinations of the frequencies of the forty modes of gamete formation. The deficiency of information is therefore much greater than with two tetrasomic loci where, from doubly digenic genotypes, we can estimate ten independent linear combinations of the frequencies of the eleven modes of gamete formation.

TABLE I 9

Deficiency matrix

\begin{tabular}{|c|c|c|c|c|c|c|c|c|c|c|c|c|c|c|c|c|c|c|}
\hline & I & . & $-I$ & . & & & $-I$ & 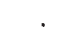 & v & . & & $\cdot$ & . & $\cdot$ & . & . & . & . \\
\hline & 2 & & & 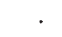 & I & . & . & I & . & . & & . & . & 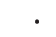 & . & . & . & I \\
\hline & 3 & . & 2 & & $-I$ & $-I$ & 3 & $-I$ & I & . & . & . & . & I & . & 1 & . & . \\
\hline & 4 & . & & . & : & . & & $-I$ & . & & . & . & . & I & . & I & . & \\
\hline & 5 & $-\dot{I}$ & $\dot{-2}$ & $-i$ & -1 & & $\dot{2}$ & -1 & $-i$ & . & & ; & : & $-i$ & $-i$ & . & $-i$ & $-I$ \\
\hline & 7 & $\mathrm{I}$ & . & & $\mathrm{I}$ & . & -3 & I & -1 & & . & . & . & & -1 & $:$ & . & : \\
\hline & 8 & & I & & . & . & & . & . & & . & . &. & 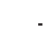 & & . & . & \\
\hline & 9 & . & & I & & - & . & . & . & & & . & . & . & & . & . & . \\
\hline & 10 & . & & . & I & . & . & . & . & & . & . & . & . & . & . & . & . \\
\hline & I I & . & & . & $-I$ & & . & . & . & & . & . & . & . & . & . & . & . \\
\hline & 12 & & . & & . & . & & & . & & . & . & . & . & . & . & . & . \\
\hline & 3 & -1 & & & . & . & . & & . & & . & . & . & I & . & & $-I$ & . \\
\hline & 4 & . & . & . & . & I & . & . & . & & . & . & . & & . & . & . & . \\
\hline & 15 & I & & - & . & . & . & & & & - & . & . & $-I$ & & $-I$ & I & \\
\hline. $\bar{c} \mathrm{r}$ & 16 & $-I$ & & . & . & . & . & $-I$ & . & & . & . & . & & . & & . & $-I$ \\
\hline I & 17 & . & & . & . & . & . & - & . & & . & . & 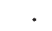 & $-I$ & . & $-I$ & . & $-I$ \\
\hline E I & 18 & I & & & $-I$ & -2 & 3 & $-I$ & I & - & & -1 & $-I$ & I & I & & I & \\
\hline$\stackrel{0}{0} \quad 1$ & 19 & & -2 & $-I$ & I & . & -3 & I & $-I$ & & . & . & . & -1 & . & $-\mathrm{r}$ & . & . \\
\hline 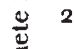 & 20 & - & & . & & . & I & 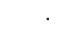 & . & & & . & . & & - & 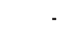 & & . \\
\hline 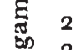 & 21 & . & & . & . & . & & . & $-I$ & & & . & . & & . & $-I$ & . & \\
\hline to & 22 & . & & . & . & . & . & . & . & - & & & . & $-I$ & I & $-I$ & I & $-I$ \\
\hline $\overrightarrow{0} \quad 2$ & 23 & . & & . & . & . & . & . & . & & . - & -1 & . & . & . & . & - & . \\
\hline$\frac{\pi}{8} 2$ & 24 & . & . & . & . & . & . & . & & & . & $\cdots$ & -1 & . & . & . & . & . \\
\hline & 25 & - & & . & & . & . & . & - & & . & $\cdot$ & & $-I$ & & • & - & - \\
\hline & 26 & . & & $\cdot$ & . & - & & $\therefore$ & - & & • & . & $\cdot$ & & -1 & - & - & \\
\hline & 27 & & . & $\cdot$ & - & • & - & I & • & & - & - & $\cdot$ & 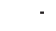 & - & - & & • \\
\hline & 28 & & & - & . & - & & I & • & & . & . & . & - & . & & $-I$ & I \\
\hline & 29 & . & - & - & . & . & . & . & I & & - & . & . & . & . & . & - & . \\
\hline & 30 & . & . & . & . & . & . & . & . & & I & . & . & . & . & . & . & . \\
\hline & $3^{I}$ & . & . & . & . & . & . & . & . & & . & . & . & . & . & . & . & . \\
\hline & 32 & . & . & . & . & . & & • & . & & • & & $\cdot$ & • & - & . & - & - \\
\hline & 33 & - & . & - & . & . & - & . & . & & . & I & . & . & . & . & . & . \\
\hline & 34 & . & & . & . & . & . & . & . & & . & . & I & . & . & & . & . \\
\hline & 35 & . & & . & . & . & . & - & . & & - & - & . & I & : & • & . & . \\
\hline & 36 & - & - & . & . & . & . & . & . & & . & - & . & . & I & & - & . \\
\hline & 37 & - & . & . & . & . & . & - & . & & • & & . & 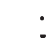 & & I & & \\
\hline & 38 & & & & . & . & . & & • & & • & - & $\cdot$ & I & $-I$ & I & $-I$ & I \\
\hline & 39 & . & . & . & . & . & - & . & . & & . & . & . & . & & & I & . \\
\hline & 40 & . & & - & & . & - & . & . & & • & . & . & . & • & . & . & I \\
\hline
\end{tabular}

\section{REFERENCES}

FISHER, R. A. 1947. The theory of linkage in polysomic inheritance. Phil. Trans., $\mathrm{B}, 233,55-87$.

FISHER, R. A. 1949. The linkage problem in a tetrasomic wild plant, Lythrum salicaria. Proc. 8th International Congress of Genetics, Hereditas, Suppl. Vol., pp. 225-233. 